\title{
A Resource Capability Model to Support Product Family Analysis*
}

\author{
Johan NIELSEN*** and Fumihiko KIMURA**
}

\begin{abstract}
In automotive industry there is a growing concern for the total economy of a manufacturing system throughout its life-cycle. In order to decrease its affect on product cost a better utilization of the manufacturing system is necessary. Product family analysis is a means to group products with common manufacturing aspects and analyze its manufacturability in a possible or existing manufacturing solution. The aim of this research is to provide support for product family analysis by enabling reasoning about resource's capability, i.e. what a resource can do and how well it can do it.
\end{abstract}

Key Words: Resource Capability, Capability Modeling, Product Family Analysis, Manufacturing

\section{Introduction}

In automotive industry there is a growing concern for the total economy of a manufacturing system throughout its life-cycle; an economy that greatly affects the cost of manufacturing an automobile. To decrease its affect on product cost better utilization of a manufacturing system throughout its life-cycle is necessary. This implies that designers and engineers must improve the reuse of manufacturing resources; not only over time, but also over product variants.

An approach to increase the reuse of manufacturing resources is to group products into families so that members of the family can share manufacturing resources. Similarly, when new members of a family are introduced the aim is to reuse the manufacturing resources with a minimum of new investments and reconfigurations.

The process of grouping products into families and understanding the consequences on the manufacturing system when new members are introduced are here called product family analysis. Product family analysis includes: i) elimination of superficial variants, i.e. same functional requirements should have the same realization; ii) knowledge of interrelationship between product functions and resource capability; and iii) finding an appropriate balance between product variation and the cost for resource (re)configuration based on this knowledge, e.g. in line

\footnotetext{
* Received 26th October, 2005 (No. 05-4231)

** Department of Precision Machinery Engineering, The University of Tokyo, 7-3-1 Hongo, Bunkyo-ku, Tokyo 113-8656, Japan. E-mail: nielsen@ cim.pe.u-tokyo.ac.jp; kimura@cim.pe.u-tokyo.ac.jp
}

(re)configuration.

Much research is being, and has been, conducted in this and related areas. In Ref.(1), for instance, an objectoriented data model to represent process planning knowledge is described. The knowledge is represented in a semantic network and used to generate alternative and optimized process plans. Lee and Saitou ${ }^{(2)}$ describe a method for designing product families that are robust to production plan variations. Alternative designs are generated and their process plan variations and manufacturing system configurations are evaluated with the objective to minimize cost while achieving efficiency. Tang et al. ${ }^{(3)}$ describe a line configuration method in the context of product families. Their method includes a genetic algorithm using, e.g., task-precedence graphs, machine reliability, machine capability, and alternative machines in order to find an optimal line design.

As indicated above, a common denominator in research within this area is an explicit or implicit model of resource capability and its relationship with product shapes. Such a model is then the base for feeding information into methods to, e.g., optimize process plans, design product families, or configure lines.

However, the conceptual relationship between product functions and manufacturing resource capability, and how to utilize the understanding of this relationship has not been formalized.

The main objective of the research presented in this paper is to provide a theoretical framework for product family analysis. This framework consists of a methodology for: i) grouping products into families; ii) eliminating superficial variants; iii) principles of product function and 
resource capability interrelationship; and iv) an information model that formalizes these principles.

The scope of this paper is, however, limited to iii) and iv) and to provide a description of the formalized model and its usage. The scope is further limited to machining processes within the automotive industry.

The research shows that reasoning about resource capability for a particular manufacturing system configuration is enabled by the developed information model. Consequently, the introduction of a new family member in an existing manufacturing line can be evaluated.

\section{Method}

The main findings of the research are based on the results from a case study involving variants of a knuckle*1. These findings were formalized in a conceptual information model as a hypothesis, from here forth called the UML-model. The hypothesis was tested by means of a second case study, an engine cylinder head, and related literature, cf. section 5 .

\subsection{Knuckle case}

The knuckle case consisted of two variants of a knuckle that, simplified, consisted of holes and planar faces. The main difference was that one variant included an ABS (Anti-lock Break System) function resulting in an additional hole at an angle to the main body. Minor differences were differences in dimensions and tolerances of holes and planar faces that were common between the both knuckles.

Although this is a simplification of the original knuckle data provided by project partners it was still representative for three different aspect that needs to be considered in product family analysis. First it represents the aspect of variance created by adding new functionality. Second it represents the aspect of variance by using different dimensions and tolerances. Third it represents the aspect of considering different features to realize; in this case holes and planar faces.

\subsection{Procedure}

The procedure used in the research of this paper was carried out in four steps:

(1) Define product family analysis; in this step product family analysis was defined and its objectives were set. Furthermore, the product family analysis procedure was described. The simplified procedure consists of:

(1.1) product design and grouping of products into families,

(1.2) generation of manufacturing system solution based on the grouping,

(1.3) evaluation of the total solution, i.e. products grouping and manufacturing system, and

*1 A knuckle is a component of the steering mechanism of a car.
(1.4) feedback of the evaluation result in order to refine the design and grouping of products.

(2 ) Identify information categories; identification of core information categories necessary to carry out product family analysis. This was carried out by analyzing the product family analysis procedure using information from the knuckle case. Three aspects — choice based on theoretical experience-were analyzed:

(2.1) Product function, i.e. information necessary to describe the product from a functional perspective

(2.2) Product manufacturing, i.e. information necessary to describe the product from a manufacturing perspective, including information about transformations and processes. Transformations were chosen because they are on a higher level of abstractions than manufacturing features, which enables a more generic way of reasoning about what is required and how to realize it.

(2.3) Resources, i.e. information necessary to describe resources including their capability and configuration. Resource information categories were identified by analyzing literature as well as machine-tool and cutting tool manuals from different vendors.

(3) Model information categories; structuring and modeling of core information categories, and adding meta level information to provide language and grammar for structuring, analyzing, and managing information.

(4) Testing; testing and evaluating the developed model by using an engine cylinder head test case as well as related literature.

(4.1) Representation of product variants both from a functional and manufacturing perspective by populating the model with information from the test case.

(4.2) Representation of a fictive manufacturing system consisting of Mazak machine-tools, including their capability.

(4.3) Manual process planning by using available information about product family and transfer-line configuration.

(4.4) Evaluation of the model's feasibility in providing necessary information for carrying out the product family analysis.

\section{Product Variants and Manufacturing Capability}

Product family analysis is here considered to be a concurrent procedure, i.e. both product aspects and manufacturing aspects are considered concurrently. Consequently, design problems are formulated based on dependencies between the two domains. These dependencies relate different aspects of products to manufacturing resources.

In the knuckle case study, essential aspects and dependencies in the context of product family analysis were identified. In Fig. 1 these aspects and dependencies are depicted conceptually; the ability to provide product func- 


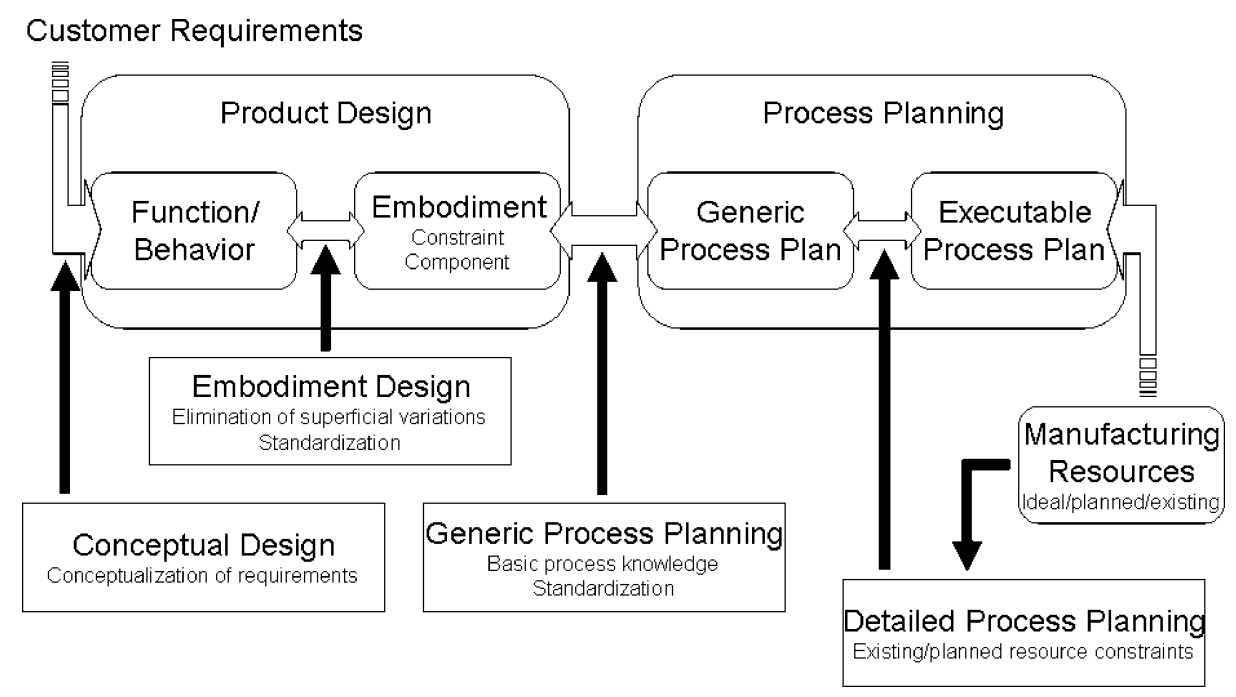

Fig. 1 Dependencies between products and manufacturing resources, adapted from Ref. (4)

tionality and desired behavior-in Product Design at the top left-depend on the capability of the manufacturing resources-below Process Planning at the top right-to realize these within specified embodiment.

In summary, there is a strong dependency between the functionality of a product and the reusability of manufacturing resources between different products. Understanding and representing these aspects and their dependency enables reasoning about the effects on a manufacturing system when introducing new functionality in a product family, or, from another point of view, the constraints on new functionality from the existing manufacturing system. Perhaps the most characteristic aspect of this dependency is the interrelation between function/behavior and manufacturing resource capability.

Dependencies for a particular case are established by mapping between the different aspects of the product- and the manufacturing domain. In the early stages product functionality is mapped to embodiment in embodiment design. Naturally there are many different mappings possible for a particular functional requirement. However, it is desirable to standardize this mapping as much as possible in order to minimize superficial variation.

By minimizing superficial variations the next mapping, from embodiment to processes, can have a higher level of commonality within a product family. That is, a higher degree of parts of the variants - sharing types of shapes and requirements - can be mapped to the same type of process. As a result, the diversification of required capability within a product family decreases. Obviously, the decreased diversification of required capability enables a mapping from processes to manufacturing resources with a higher degree of resource sharing and reusability.

\section{Model Concepts}

The findings from the knuckle case study have been used to conceptualize aspects in, and dependencies between, the product and manufacturing domain. The conceptualization has been formalized by the use of UML class diagrams. The intension of this section is to describe parts of this formalization that are of importance for representing resource capability.

Taxonomy: A taxonomy mechanism is used to classify objects such as features, processes, and capability. This mechanism is the point of reference for reasoning about these objects. The main structure of the taxonomy mechanism is hierarchical. However, other relationships may also be represented, e.g. constraint between two nodes in a hierarchy.

Constraint: A generic constraint mechanism is included that enables constraining of any object. In addition, constraints between any two objects can also be represented. The intention of the constraint itself is to enable any type of constraint representation, e.g. textual, numerical, and mathematical.

Capability: Capability is the means to describe what, and how well, a resource can do something, and is represented by a capability profile, cf. Fig. 2 . The capability profile represents capability common to any process, such as maximum allowed workpiece size and table load, as well as capability specific to a particular type of process, such as a resource's accuracy of diameter when machining a hole. The former is represented by associating properties to a capability profile whereas the latter is represented by associating properties to a process capability.

The process capability is dependent on the context in which a resource is used, i.e. if a machine-tool is used for machining a hole, a slot, or a planar face. The context or what a resource can do and how it does it is represented 


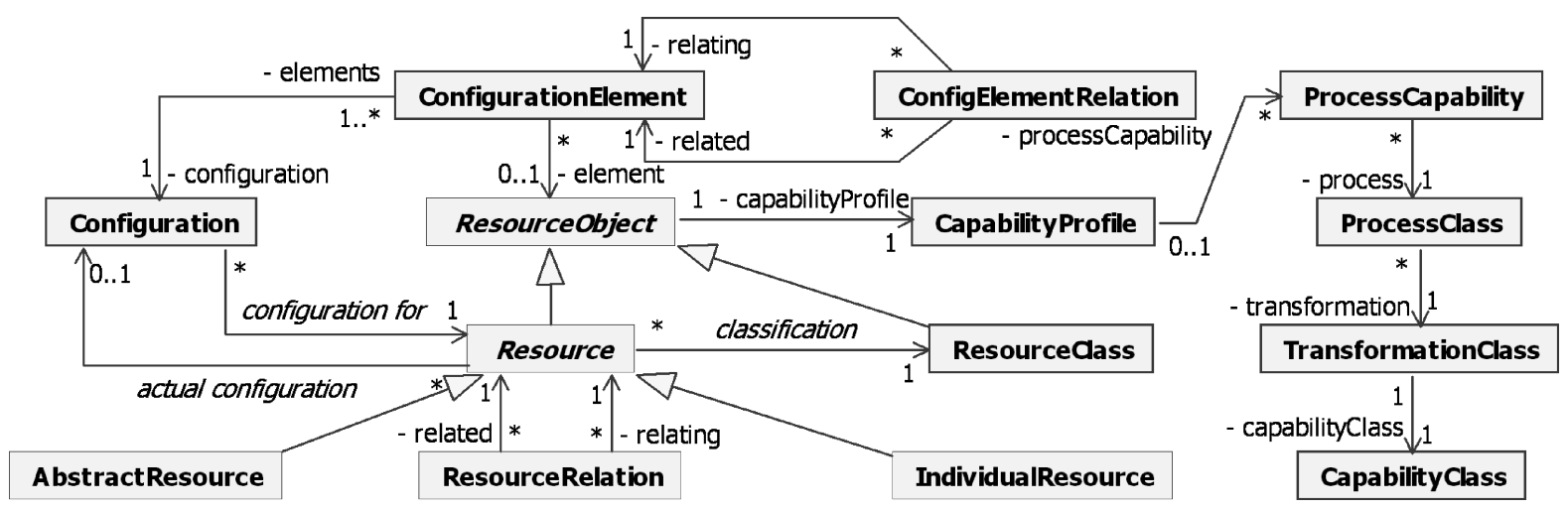

Fig. 2 The mechanism to represent resources, configurations, and their capability

by a transformation class and a process class respectively. For instance, a hole-transformation may be realized by a twist-drilling type of process. Consequently, by separating the type of transformation from the type of process, reuse of transformation definitions between different process classes is enabled.

However, enabling this reuse was only one of two reasons for introducing a separation between transformation and process. The other reason was that the type of transformation defines the type of key characteristics that will be represented by a process capability, whereas the type of process merely modifies their values. For instance, the types of key characteristics for a hole-transformation are independent of the type of process, but the use of twistdrilling would result in surface finishes of 3.125-12.5 $\mu \mathrm{m}$ whereas boring would result in surface finishes of $0.8-$ $12.5 \mu \mathrm{m}^{(5)}$.

The set of key characteristic types is represented by a capability class that is associated with a transformation class. Consequently, the capability class defines the type of properties that must be associated with a process capability in a particular transformation context.

Resources and configurations: Resources are non consumable things providing some capabilities that can be used to realize a product. Such resources are here represented in three different ways; as classes of resources, as conceptual (abstract) resources, and as individual resources. Figure 2 depicts the mechanism to represent resources and their configurations.

The resource class is used to define the type, or class, of a particular resource. At some level in a resource class structure a, e.g., 3-axes mill resource class may be found. This class may be further decomposed into several different atomic classes. One of theses atomic classes may be a Mazak UN600V, which is a 3-axes vertical mill. In this case the Mazak UN600V represents a concrete class of resources whereas the 3-axes mill class represents resources on a higher level of abstraction.

Classified resources can then be represented as abstract or individual resources. The main difference be-

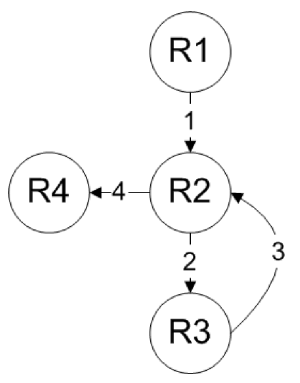

Fig. 3 An example manufacturing system configuration

tween these is that an abstract resource represents a concept whereas an individual resource represents a physical resource in a facility. As an example of the difference is the concept of using a 3-axes vertical mill for face milling and using a particular physical Mazak UN600V, which is a type of 3-axes vertical mill, for the same process. The first would be represented by an abstract resource, classified as a 3-axes vertical mill, while the latter would be represented by an individual resource, classified as a Mazak UN600V.

Despite the difference in the level of abstraction, both abstract and individual resource can have configurations associated. A configuration defines a valid set of resource objects that can be combined in order to realize the resource. For instance, consider the 3-axes vertical milling machine-tool concept above for face milling. A configuration could say that a milling type cutting tool needs to be mounted in order for any 3 -axes vertical mill to provide the process.

Furthermore, configurations can also specify, e.g., line configurations that includes constraints on the flow of material between the different resources. Figure 3 exemplifies the sequential flow constraint between resources, i.e. the flow of material is allowed in the direction of the arrows between the resources-e.g. flow 1 between R1 and R2.

As depicted in Fig. 3, the mechanism in the model also allows for representing more complex sequential configurations so that particular resource can be reused 
later on in the flow, e.g. resource $\mathrm{R} 2$ which is used directly after resource R1 and again after resource R3. In addition to sequential flows, parallel flows as well as mountings/assemblies - e.g. tools and fixtures mounted on a machine-tool—can be represented.

In summary, the resource and configuration mechanism is a means to represent concepts and individual resources and their configurations.

Process plan: The process plan mechanism is a means to represent a set of instructions of how to achieve a set of transformations. This can be represented at different levels of process plan abstractions, i.e. from generic process plans to executable process plans. At each level different types of relations between processes can be represented, e.g. precedence, sequence, alternative, and decomposition.

\section{Model Testing: An Engine Cylinder Head Case Study}

In order to test that the model can be used to represent data for product family analysis an engine cylinder head case study was conducted. The chosen scenario for the case study was to evaluate if an engine cylinder head could be manufactured in an existing cylinder head's manufacturing line. Hence, the model's ability to represent necessary data for such an evaluation was tested.

A limitation in the case study was that only engine cylinder head data was available and, thus, a fictive line configuration had to be used. This fictive line was made up of Mazak machine-tools with data from their machinetool specifications. Naturally, these specifications did not contain any process capability data. Consequently, it was necessary to create this data as well; using sources such as Refs. (5) and (6) to make it realistic.

Prerequisites for the chosen scenario were that a generic process plan for an engine cylinder head had already been created, a configuration of the current line already existed, and, of course, that necessary taxonomy and resource data was available. Note also that a generic process plan is here considered to be made up of processes with specified process classes and required capability, as well as precedence constraints between the processes.

\subsection{Case study objects}

Engine cylinder heads: The case study object was an engine cylinders head. From the engine cylinder head some parts, such as the hydraulic lash adjusters (HLAs), and spring seats were chosen as study objects, cf. Fig. 4. These parts were manufactured in 35 processes accessed from the top view (left in Fig. 4), the bottom view (right in Fig. 4), and from the front view (facing downwards in Fig. 4).

Since available information only concerned one cylinder head a fictive variant had to be created. With the available information as a base a second smaller engine cylinder head with, overall, tighter tolerance were created. Hence, the min differences between the engine cylinder heads were dimensions, weight, and tolerances.

The second and smaller engine cylinder head also needed less manufacturing processes with slightly differences in sequential constraints. That is, it had less manufacturing steps and the steps could be arranged in a slightly different sequence.

Processes: A library of typical machining processes was also created. The library was mainly based on theoretical experience, i.e. literature studies. It was also the base for describing the capability of the resources in the transfer-line.

Resources: The resources used were Mazak machine-tools configured in a transfer-line type manufacturing system according to Fig. 6 below. Two differ-

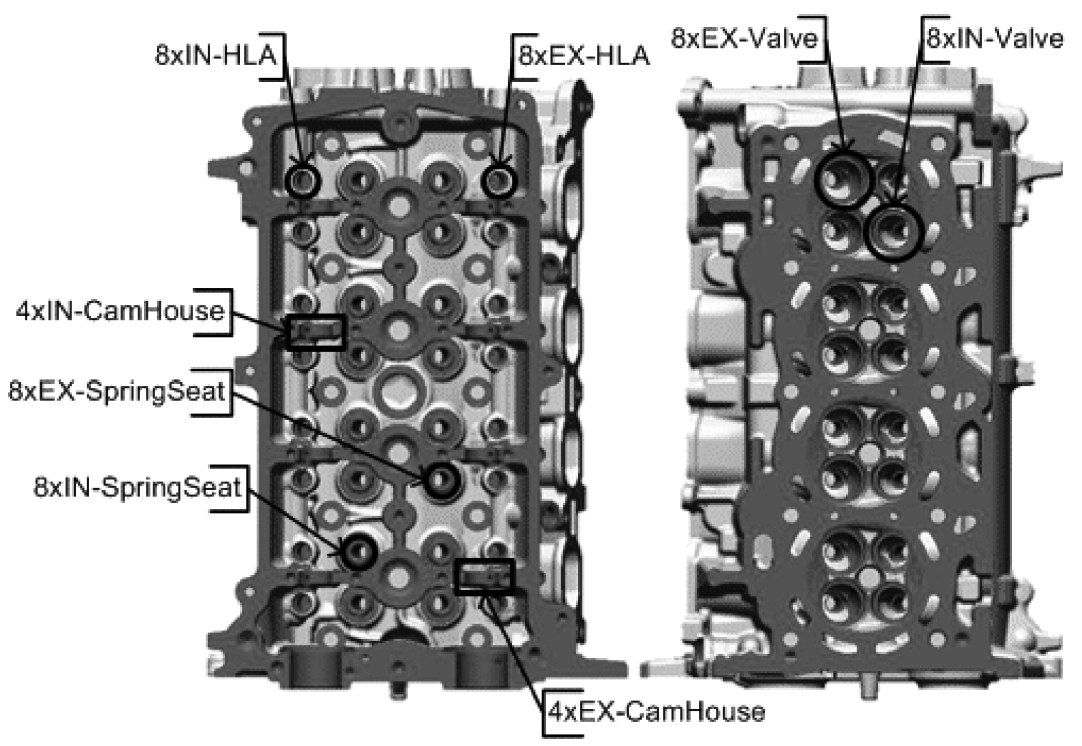

Fig. 4 The case study object: an engine cylinder head 
ent types of machines were used in the line, the Mazak UN600V and Mazak UN600H. The first is a 3-axes machine-tool and the latter a 4-axes machine-tool, both capable of several different types of processes such as drilling and milling.

For the purpose of the test case some significant differences between the two machine's capability were introduced, although in reality by comparing the both machines specification brochures no significant differences could be identified. The 3-axes machine was chosen to be capable of smaller, lighter, and lower quality manufacturing than the 4-axes machine. This means limiting some of the processes from being carried out by the 3-axes machine.

\subsection{Procedure}

The chosen procedure for evaluating if the engine cylinder could be manufactured in an existing line was: i) select a valid process sequence according to the process plan constraints, ii) allocate processes to resources according to sequence constraints, iii) evaluate if a resource is capable in terms of required capability and tool access directions (TADs).

\subsection{Process sequencing}

As mentioned before, a generic process plan was a prerequisite, and consisted of processes, precedence constraints, and process classes. A simplified example of a generic process plan for the engine cylinder head is depicted to the left in Fig. 5.

When selecting a process sequence the generic process plan is used to decide which processes that are eligible for a particular position in the sequence. When a process is selected for a position, its constraints are virtually removed and the eligible process queue is updated. Hence, a new set of eligible processes are available for selection at the next position.

The selection of a process from the eligible process queue can be based on different criteria. For instance, one criterion could be to remove as many precedence constraints as possible in each selection, and another criterion could be to select the process that is most similar to the previous one.

\subsection{Resource allocation}

In Fig. 6 the fictive line configuration is depicted. The resource at the lower right represents the line, here named Line\#8. It identifies a configuration that specifies a set of resources as part of the configuration, represented as configuration elements (CE). The sequence constraints $(\rightarrow)$ between configuration elements represent the sequence between resources in Line\#8.

Now, consider the two processes depicted in Fig. 5. If the IN-HLA face process allocates resource $B \# 3$ and the $I N$-HLA hole process allocates resource $A \# 2$, then there is a conflict between the precedence constraints in the process plan and the sequence in the configuration. The process plan specifies that the IN-HLA face should be carried out before the IN-HLA hole, whereas the suggested allocation to resources specifies that the IN-HLA face should be carried out after the IN-HLA hole.

However, sequence conflicts can easily be avoided by

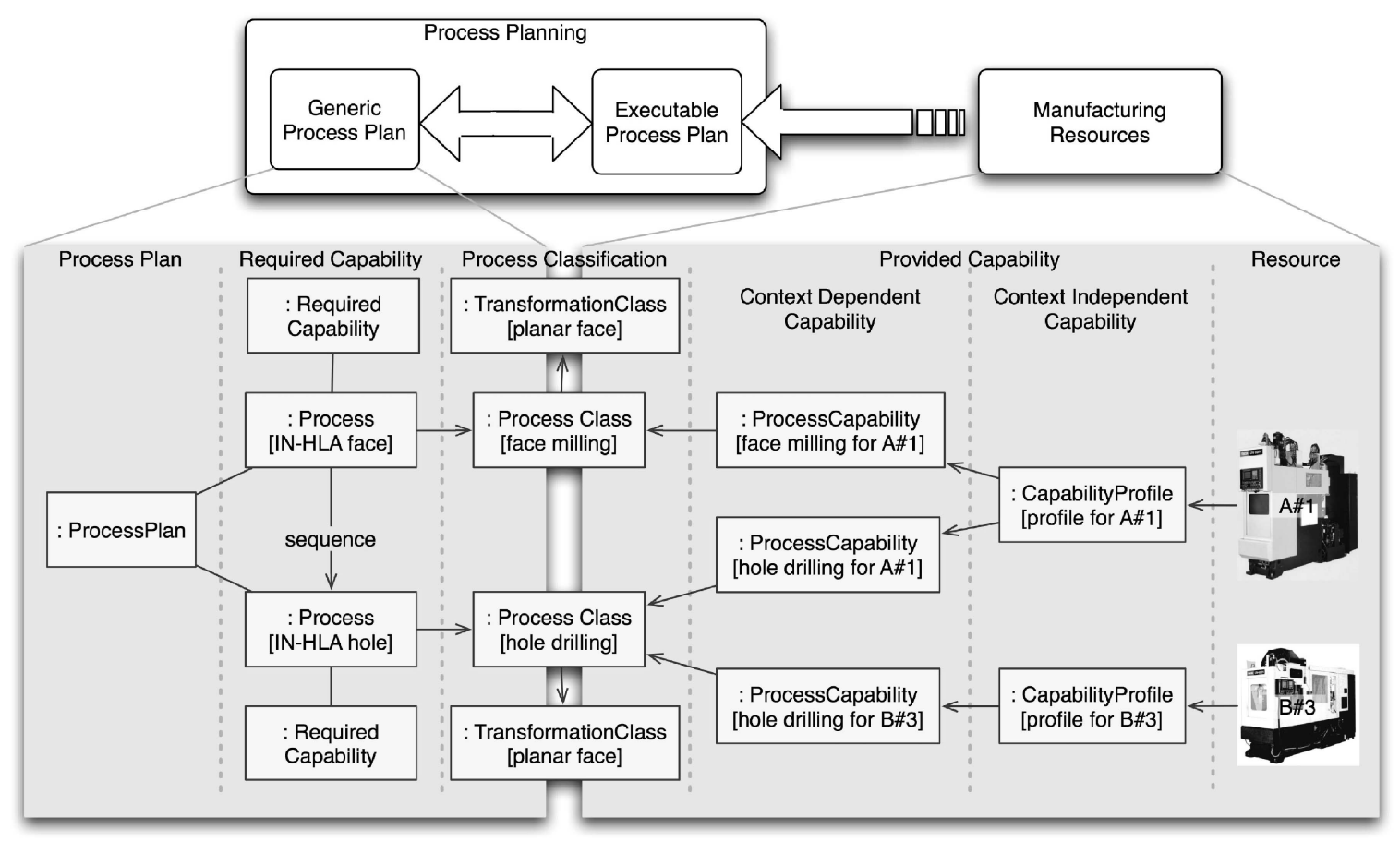

Fig. 5 Schematic overview of an instantiated process plan with its required capability, and of instantiated resources and their provided capability 


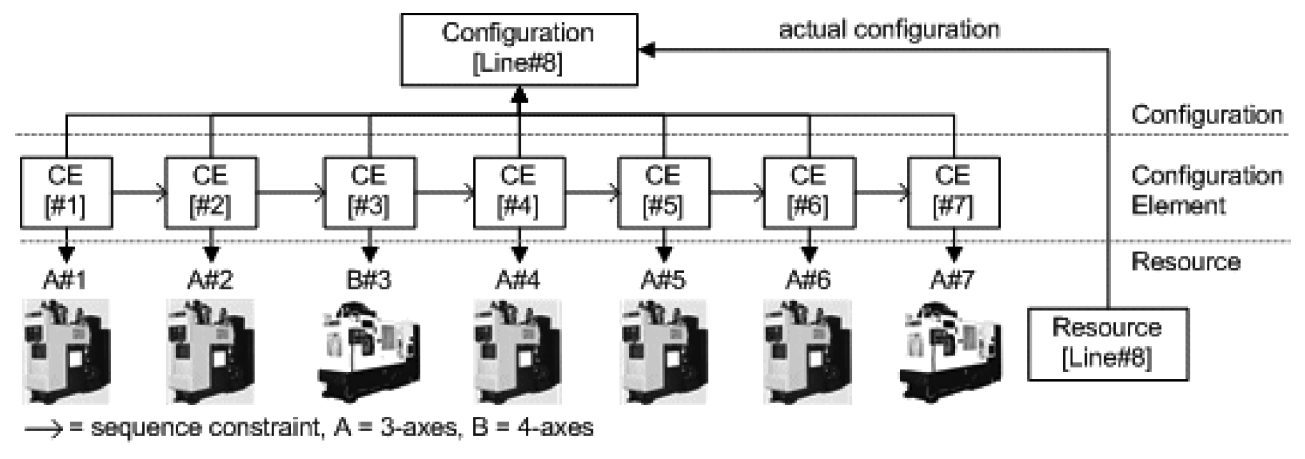

Fig. 6 Schematic overview of an instantiated line configuration

using the line configuration data in combination with the process sequence (or the eligible process queue) data. For example, by allocating the first set of processes (according to the process sequence) to the first resource (according to the line configuration), then the second set of processes to the second resource, and so on, the sequence conflicts can be avoided.

\subsection{Evaluating resource capability}

The evaluation of an allocated resource's capability is carried out in three steps. First, the process class of a process is identified. Second, the allocated resource's provided capability is retrieved by extracting the properties of its capability profile and its process capability for the particular process class. Third, the provided capability is compared with the required capability.

Using the example in Fig. 5 this means that for the $I N$ HLA face process the face milling process class is identified. The provided capability would then be the properties of capability profile for A\#1 and process capability face milling for A\#1.

The properties of a provided capability follow the format nominal, accuracy, capability index ${ }^{* 2}$. These are then compared to the properties of the required capability following the same format. The required diameter of the $I N$ $H L A$ hole process is $12.0 \pm 0.1 \mathrm{~mm}$ with $C_{\mathrm{pk}}=1.6$. This must be matched with a provided capability with the name diameter and with a nominal value of $\geq 12.0 \mathrm{~mm}$, an accuracy value of $\leq 0.1 \mathrm{~mm}$ and a capability index of $\geq 1.6$ in order for the resource to be considered capable. Both resources $A \# 1$ and $B \# 3$ in Fig. 5 are capable since allowed diameter $\leq 130 \mathrm{~mm}$ (based on maximum allowed tool diameter) and they both have an accuracy $\geq 0.1 \mathrm{~mm}$ (i.e. $0.1 \mathrm{~mm}$ is their best accuracy) for $C_{\mathrm{pk}} \geq 1.6$.

\subsection{Evaluating tool access direction}

In order to evaluate if a resource (machine-tool) is capable of accessing all the tool directions required by a set of processes for a particular setup two types of data are needed. The first type of data is the tool access directions (TADs) of the set of processes for a particular setup. The

\footnotetext{
${ }^{* 2}$ Here $C_{\mathrm{pk}}$ is used as a capability index.
}

second type of data is the axes data of the used machinetool, i.e. the number of axes and axes strokes.

A TAD is represented as a property of a process' required capability whereas axes data is represented as a list of properties of a resource's capability profile. Hence, once a set of processes allocates a resource, the necessary data can be retrieved and the resource's capability of accessing all the TADs can be evaluated.

\section{Discussion and Conclusions}

The UML-model described in section 4 was able to represent data about the engine cylinder head and resources necessary in order to evaluate the manufacturability of the engine cylinder head in an existing line. The manufacturability evaluation included evaluation of process and line sequencing as well as individual resource's capability, including tool access directions.

Since only product data was available for the case study a fictive line was developed, and data about individual resources was collected from different literature sources. Nevertheless, the ability of the UML-model to represent process plans (including required capability such as tool access directions and accuracy), resource configurations, and resource capability (including axes data and accuracy) was corroborated.

The above conclusions indicate that the feeding of product family information and the generation and optimization of transfer-lines can be automized, because the required information is provided by the model. Especially the meta level information that enables reasoning about resources and their capability in relation to product requirements. By a demonstrator software application for configuration and optimization of transfer-lines, based on the findings by Ref. (3), corroborating this statement has already been implemented and tested.

This demonstrator shows what the result of this paper implicates, that by structuring information and providing the correct meta level information line configuration and optimization can be automated. This would eliminate error prone manual procedures both in the transferring of product family information from design to manufacturing 
system development as well as in the actual manufacturing system development procedure.

Not only would this decrease the cost of developing a manufacturing system but most likely also decrease the overall cost of using the manufacturing system throughout its life. The reason for this is that the models also enables the continuous update of capability data from the actual usage of the resources in the factory. Hence, by always having a correct understanding of what the resource can do it will be possible to utilize their capability in more efficient way.

Models representing capability can also be found in Refs. (1) and (7). Both similarities and differences between these models and the UML-model can be identified. The similarities are that all the models define capability in relation to process classes (or similar).

In Ref. (1) these process classes also represent the actual capability and, thus, represent capability common for all resources that can provide that type of process. In contrast, the model in Ref. (7) represents capability that is specific for an individual resource. The UML-model enables both types of capability representations.

An interesting characteristic of the UML-model, which is not part of Refs. (1) and (7), is that in combination with the capability representation it also provides a mechanism for representing line configurations. This is an important aspect in order to support product family analysis.

In Refs. (2) and (3) manufacturing system configurations are used. However, they are mainly as a result of a configuration/optimization procedure, and not used as a constraint on process planning.

In conclusion, the UML-model enables reasoning about resource capability for a particular manufacturing system configuration. Consequently, product family analysis, such as the evaluation of the manufacturability of a new family member in an existing manufacturing line, is supported. In addition, a structured method in combination with the information provided by the UML-model can achieve an overall lower manufacturing cost by eliminating the need for error prone manual design processes as well as a better utilization of resource.

\section{Acknowledgment}

This research is performed as a part of the project Life Cycle Oriented Design of Flexible and Agile Production Systems (LicoPro) within the IMS program supported by the Ministry of Economy, Trade and Industry (METI), Japan. The authors would like to extend their appreciation to all the project members: Toyota Motor Corporation, Yamazaki Mazak Corporation, Mitsubishi Electric Corporation, Toyota Central Research and Development Laboratories, and Toyota Tsusho Corporation. Johan Nielsen is supported by the Postdoctoral Fellowship for Foreign Researchers by Japan Society for the Promotion of Sciences (JSPS).

\section{References}

( 1 ) Sormaz, D.N. and Khoshnevis, B., Process Planning Knowledge Representation Using an Object-Oriented Data Model, The International Journal of Computer Integrated Manufacturing, Vol.10, No.1-4 (1997), pp.92104.

( 2 ) Lee, B. and Saitou, K., Design of Part Family Robustto-Production Plan Variations Based on Quantitative Manufacturing Evaluation, Research in Engineering Design, Vol.13, No.4 (2002), pp.199-212.

( 3 ) Tang, L., Yip-Hoi, D.M., Wang, W. and Koren, Y., Concurrent Line-Balancing Equipment Selection and Throughput Analysis for Multi-Part Optimal Line Design, The International Journal for Manufacturing Science \& Production, Vol.6, No.1-2 (2004), pp.71-82.

( 4 ) Kimura, F. and Nielsen, J., A Design Method for Product Family under Manufacturing Resource Constraints, CIRP Annals-Manufacturing Technology-, Vol.54, No.1 (2005), pp.139-142.

( 5 ) Halevi, G. and Weil, R.D., Principles of Process Planning, (1995), Chapman \& Hall.

( 6 ) Bralla, J.G., Design for Manufacturability: Handbook, (1998), McGraw-Hill.

( 7 ) ISO/TC184/SC5, Manufacturing Software Capability Profiling for Interoperability-Part 2 (ISO/DIS16100-2), International Organization of Standardization, (2002). 